\title{
Design of the Variable Inclination Continuous Transverse Stub Antenna Based on Rectangular Grating Slow-Wave Structure
}

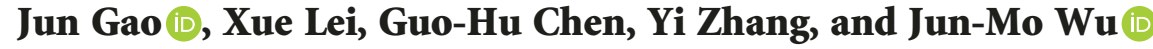 \\ National Digital Switching System Engineering and Technological R\&D Center, Zhengzhou 450002, China \\ Correspondence should be addressed to Jun Gao; gjaoun121@163.com
}

Received 25 October 2017; Revised 25 January 2018; Accepted 5 February 2018; Published 2 April 2018

Academic Editor: Paolo Burghignoli

Copyright ( 2018 Jun Gao et al. This is an open access article distributed under the Creative Commons Attribution License, which permits unrestricted use, distribution, and reproduction in any medium, provided the original work is properly cited.

\begin{abstract}
In order to solve difficulty in designing nonlinear slow-wave structure (SWS) of the variable inclination continuous transverse stub (VICTS) antenna, we combine the study of the semiopen rectangular grating SWS in the PPW with equivalent circuit theory of leaky-wave antenna to propose a theoretical model for SWS of VICTS element. The computations that the propagation characteristics of VICTS element are calculated by the theoretical model. The results are consistent with the fullwave simulation, which prove the validity and accuracy of the theoretical model. Lastly, by using the theoretical model, an approach for nonlinearly designing SWS is introduced and carried out. The approach improves the gain of the VICTS antenna $0.4 \mathrm{~dB}$.
\end{abstract}

\section{Introduction}

VICTS antenna is a novel low-profile antenna. Different functional layers of the antenna are rotated in plane to steer variation of elevation, azimuth, and polarization angles of the beam. Compared with an active phased array antenna, feed structures of this low-profile antenna are simpler [1]. It possesses high gain and flexible beam steering without $\mathrm{T} / \mathrm{R}$ component employed. Compared with reflector antenna, the height of existing VICTS antenna is only $10 \mathrm{~cm}$ at $\mathrm{Ku}$ band, and it is a prospect for communications on the move. In addition, the low interference of adjacent satellites and tunable power spectral density of beam, due to the cross distribution of sidelobe, greatly improve the satellite bandwidth efficiency. VICTS antenna has wide application prospect in satellite mobile communication.

VICTS antenna is developed from continuous transverse stub (CTS) antenna [2-4] and it is a typical frequencyscanning and leaky-wave antenna. The antenna structure is shown in Figure 1. When rotating the transverse stub of the CTS antenna relative to the feed structure, CTS antenna evolves into VICTS antenna and possesses beam scanning capability. In order to achieve large scanning angle and suppressing the grating lobes in beam scanning, SWS should be designed in the parallel-plate waveguide (PPW), for example, dielectric-filled PPW which reduces waveguide wavelength. However, dielectric slab produces losses and reduces the gain of the antenna system. Therefore, a designer uses a rectangular grating metal structure instead of the dielectric slab. The periodic disturbances of rectangular grating excite spatial harmonics and fast-wave components of harmonics radiate $[5,6]$.

Figure $1(\mathrm{c})$ is the element structure in VICTS array. The changing height corrugations on the lower parallel plate are rectangular gratings. The antenna radiates through the upper plate named CTS layer, which is composed of slots and stubs. The length of the element in the $\mathrm{Y}$ direction is much greater than the wavelength. The element is cascaded in the $\mathrm{X}$ direction and is fed by line source which provides quasi-TEM wave excitation arranged on one side of the PPW.

In order to efficiently control the main lobe width and sidelobe level of VICTS antenna, the height of the PPW tends to be nonlinear as energy propagating. However, when the quasi-TEM wave propagates in the slope-shaped PPW, the propagation constant nonlinearly varies with the height of the PPW and it causes the gain of VICTS antenna to 


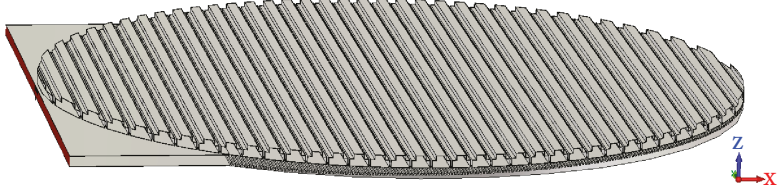

(a)

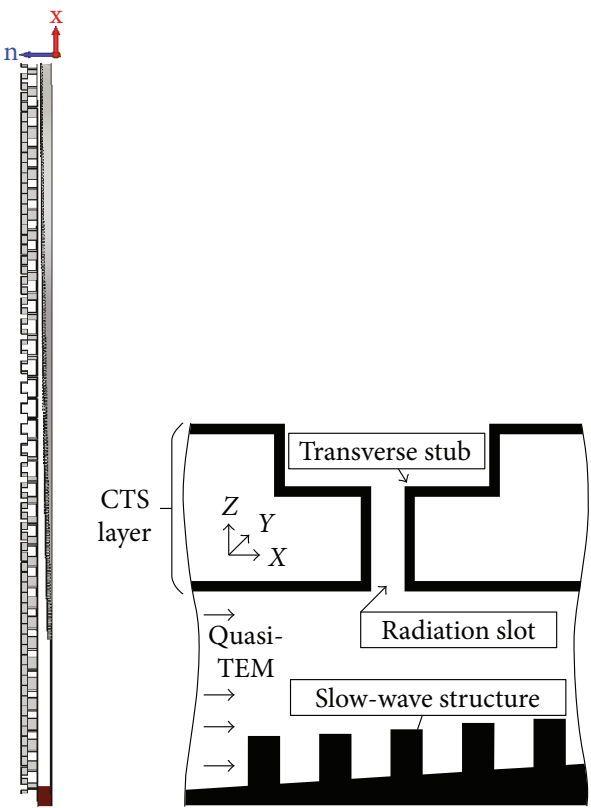

(c)

FIgURE 1: Radiating array of VICTS antenna: (a) 3D view, (b) side view, and (c) side view of VICTS element.

deteriorate. Therefore, the propagation characteristic of the element in VICTS array has to be analyzed. But it has not been extensively addressed in semiopen SWS with slots cut, and there is no such reference to guide how to design nonlinear rectangular grating structure in VICTS antenna either. Thus, in this paper, we combine field theory and circuit theory to study the dispersion equation of VICTS elements. Then, by applying this equation, we put forward an approach to adjust the rectangular grating structure nonlinearly to keep the propagation constant still.

\section{Research on Propagation Characteristics of Rectangular Grating SWS}

Research on the propagation characteristic of VICTS element originates from that of the SWS-loaded waveguide. Eliminating the slots on the upper plate, VICTS element becomes semiopen rectangular grating SWS, that is, naming as unperturbed structure in this paper. The study of this semiopen rectangular grating SWS can be acquired from [7-9] which studies a similar structure. As shown in Figure 2, energy propagates in the $\mathrm{X}$ direction. The structure is long enough to neglect truncation in the $\mathrm{X}$ direction and arranges perfect magnetic boundary in the $\mathrm{Y}$ direction. $h w g=(b+h)$ is the height of the PPW, the width, period, space, and height of the rectangular grating is $a, p, s$, and $h$, respectively.

We adopt the field-matching method to analyze fields in an unperturbed structure whose height is constant and the height of rectangular grating in it is constant as well. The structure can be divided into two parts: The first part (I) is the interaction region, and the second part (II) is the rectangular grating region. According to longitudinal field method, each field component can be derived from $H_{y}$. In region $\mathrm{I}, H_{y}^{\mathrm{I}}$ is decomposed into summation of the infinite Bloch components, then substitute the summation into the Laplace equation. When slow wave is propagating in the region I, we can solve $H_{y}^{\mathrm{I}}$ by

$$
H_{y}^{\mathrm{I}}=\sum_{m=-\infty}^{+\infty} A_{m}^{\mathrm{I}} \cosh \left(\gamma_{m}^{\mathrm{I}} z\right) e^{-j \beta_{m} x}
$$

where

$$
\begin{aligned}
& \beta_{m}=\beta^{\text {unp }}+\frac{2 m \pi}{p}, \\
& \gamma_{m}^{\mathrm{I}}=\sqrt{\left|\beta_{m}^{2}-\beta_{0}^{2}\right|} .
\end{aligned}
$$

$A_{m}^{\mathrm{I}}$ is the amplitude of the $m$ th harmonics, $\beta_{m}$ is the propagation constant of the $m$ th harmonics in the $\mathrm{X}$ direction, $\beta^{\text {unp }}$ is the propagation constant in the unperturbed PPW. $\beta_{0}$ is the propagation constant in free space. $m=0$ and $m=-1$ harmonics have the greatest contribution to the summation of the spatial harmonics, and the dispersion equation of the unperturbed structure converges quickly. Therefore, the truncation that $m= \pm 3$ is adopted. The truncation error is less than $10^{-6}$ [7].

In region $\mathrm{II}$, the space in the $\mathrm{X}$ direction is closed. $H_{y}^{\mathrm{II}}$ can be decomposed into summation of the infinite standing wave:

$$
H_{y}^{\mathrm{II}}=\sum_{n=0}^{+\infty}\left[A_{n}^{\mathrm{II}} \cos \left(\gamma_{n}^{\mathrm{II}} z\right)+B_{n}^{\mathrm{II}} \sin \left(\gamma_{n}^{\mathrm{II}} z\right)\right] \cos \left(\beta_{n} x\right)
$$




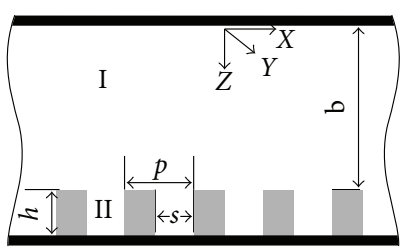

(a)

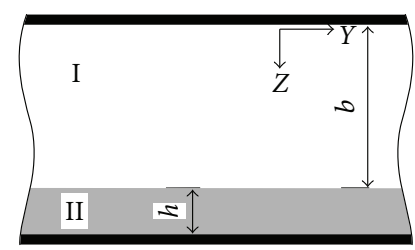

(b)

Figure 2: Diagram of unperturbed structure: (a) transverse section view and (b) longitudinal sectional view.

where

$$
\begin{aligned}
& \beta_{n}=n \frac{\pi}{s} \quad(n=0,1,2, \ldots), \\
& \gamma_{n}^{\mathrm{II}}=\sqrt{\left|\beta_{n}^{2}-\beta_{0}^{2}\right|},
\end{aligned}
$$

where $A_{n}^{\mathrm{II}}, B_{n}^{\mathrm{II}}$ is the amplitude of the $n$th harmonics and $\beta_{n}$ is the propagation constant of the $n$th harmonics in the $\mathrm{X}$ direction. The following boundary conditions are applied:

(1) Metal surface where $z=b+h$ satisfies the condition $E_{x}^{\mathrm{II}}=0$.

(2) At the interface, $z=b$ between I and II satisfies the condition

$$
\begin{aligned}
& H_{y}^{\mathrm{I}}=H_{y}^{\mathrm{II}}, \quad 0<y<s, \\
& E_{x}^{\mathrm{I}}=\left\{\begin{array}{cc}
E_{x}^{\mathrm{II}}, & 0<x<s, \\
0, & s<x<p .
\end{array}\right.
\end{aligned}
$$

We can eliminate amplitude coefficients of the harmonics by substitution. As a consequence, the dispersion (6) of the unperturbed structure in the PPW is derived.

$$
\frac{1}{\tan \left(\gamma_{0}^{\mathrm{II}} h\right)}-\sum_{m=-\infty}^{+\infty} \frac{4 \gamma_{0}^{\mathrm{II}} \sin ^{2}\left(\beta_{m} s / 2\right)}{\gamma_{m}^{\mathrm{I}} \beta_{m}^{2} p s \tanh \left(\gamma_{m}^{\mathrm{I}} b\right)}=0
$$

Equation (6) establishes the relationship between $\beta^{\text {unp }}$ and the parameters of unperturbed structure. In order to ensure sufficient computational accuracy, free space wavelength $\lambda_{0}>4 \mathrm{~h}$. Besides, when $b \gg \lambda_{0}$, region I can be approximate to vacuum and the condition meet the definition in [8]. When $b \ll \lambda_{0}$, there are higher-order spatial harmonics been neglected in (6) which has been studied in [9].

\section{Research on Propagation Characteristics of Slow-Wave Structure with Slots Loaded}

From equation (6), it can be seen that the parameters of VICTS element are numerous and interrelated. In the previous section, because of the approximate assumption that the perturbation for the field can be superimposed, slots have been neglected in the unperturbed structure. In this section, based on the unperturbed structure, we can easily analyze the perturbation of periodic slots in the perturbed structure shown in Figure 3 by applying leaky-wave circuit theory. The slots, stubs, and SWS of the perturbed structure in Figure 3 is periodic.

Since SWS is a dispersive system, VICTS antenna is a frequency-scanning antenna. When the quasi-TEM feed does not rotate relatively to the CTS layer, beam scanning in the XOZ plane. The scanning angular position $\theta$ is defined by

$$
\sin \theta=\frac{\beta_{-1}}{\beta_{0}}
$$

Because of the perturbation of periodic slots, the antenna mainly radiates -1 th harmonic (fast wave). In order to obtain the propagation constant $\beta_{-1}$ of -1 th harmonic, we combine the dispersion equation of unperturbed structure with leakywave circuit theory to research the propagation characteristics of the structure shown in Figure 3. The equivalent circuit model of VICTS array is derived from [10] and is shown in Figure 4: PPW filled with rectangular grating is equivalent to the transmission line whose characteristic impedance is $Z_{c}$ and propagation constant of the fundamental mode is $\beta^{\text {unp }}$. Each slot is equivalent to the impedance $Z_{s}$ connected in series on the transmission line. By means of Bloch theory, the complex propagation constant $k_{x}^{B}$ of the perturbed structure is obtained

$$
\begin{aligned}
\cosh \left(k_{x}^{B} d\right) & =\cos \left(\beta^{\text {unp }} d\right)+\frac{j}{2} \bar{Z}_{s} \sin \left(\beta^{\text {unp }} d\right), \\
k_{x}^{B} & =\alpha_{x}^{B}+j \beta_{-1} .
\end{aligned}
$$

$\alpha_{x}^{B}$ is the attenuation coefficient. $Z_{c}$ is quite close to the characteristic impedance $\eta_{0} h w g / W, \eta_{0}$ is free space impedance, $W$ is the width of PPW. $\bar{Z}_{s}$ is $Z_{s}$ normalized to $Z_{c}$. After obtaining $\beta_{-1}$, the equivalent permittivity $\varepsilon_{e}$ of element can be calculated by

$$
\varepsilon_{e}=\left(\frac{\beta^{-1}+(2 \pi / d)}{\beta_{0}}\right)^{2} .
$$

Take a circular VICTS antenna with a diameter of $700 \mathrm{~mm}$ shown in Figure 5 as an example. Its element is periodic just as shown in Figure 3. The excitation source is on one side of PPW and the electric field direction is perpendicular to the upper plate. The electromagnetic model is established in the full-wave simulation software CST. Wave port is used to excite the antenna, then patterns of VICTS antenna are obtained as shown in Figure 6. Meanwhile, $\varepsilon_{e}$ and beam 


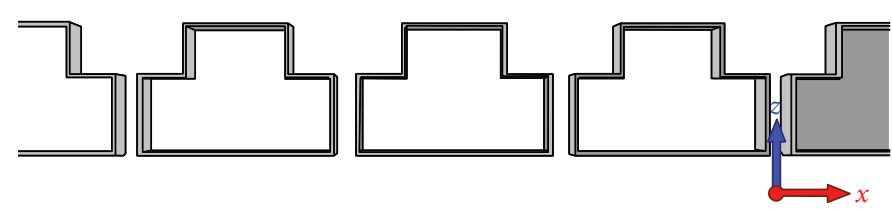

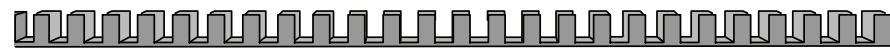

Figure 3: Side view of the simulation model of the perturbed structure. Four elements are cascaded in the $\mathrm{X}$ direction and the element is periodic.

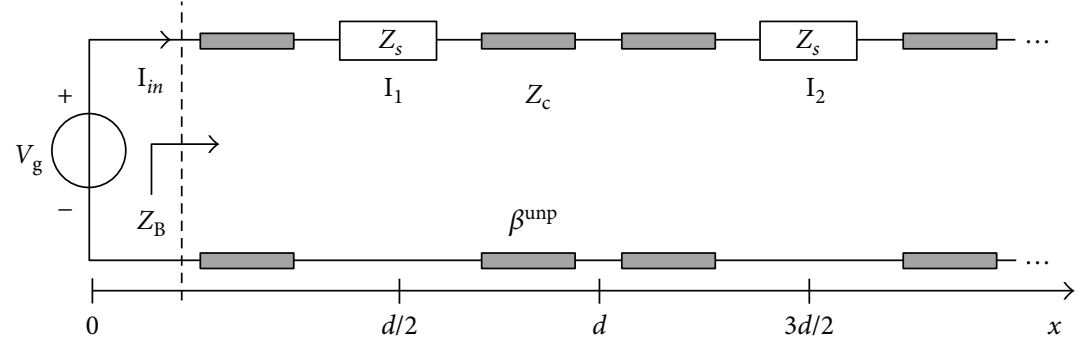

FIGURE 4: The equivalent circuit model of VICTS antenna.

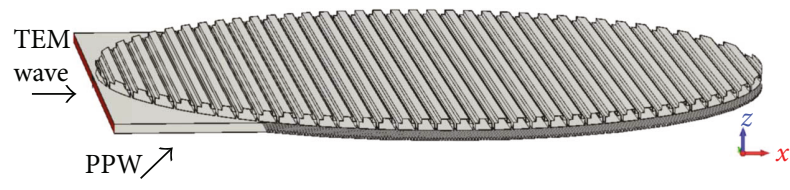

FIGURE 5: Prototype of $700 \mathrm{~mm}$ circular-aperture VICTS antenna. Based on the periodic element shown in Figure 3.

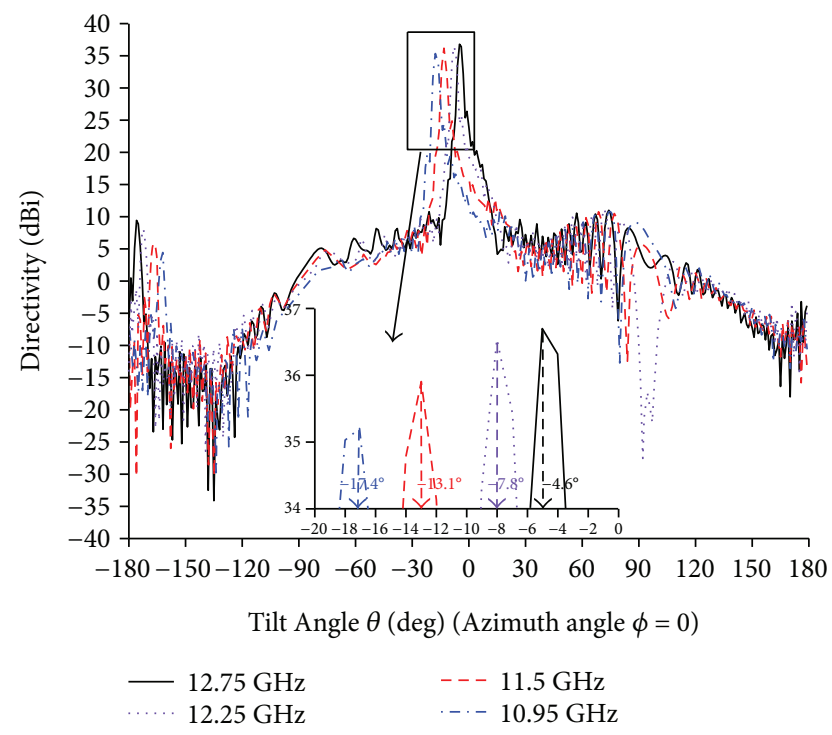

Figure 6: The full-wave simulation patterns of $700 \mathrm{~mm}$ circularaperture antenna model when relative rotation between CTS layer and SWS is zero.

direction $\theta$ under different operating frequencies are calculated by (6-10). The data of $\varepsilon_{e}$ comes from 10 cascaded elements similar to Figure 3. The comparison of computation between the theoretical model $(6-10)$ and the full-wave
TABLE 1: Comparison of theoretical model and full-wave simulation.

\begin{tabular}{|c|c|c|c|c|c|c|}
\hline \multirow{2}{*}{$\begin{array}{l}\text { Frequency } \\
(\mathrm{GHz})\end{array}$} & \multicolumn{2}{|c|}{$\begin{array}{l}\text { Full-wave } \\
\text { simulation }\end{array}$} & \multicolumn{2}{|c|}{$\begin{array}{c}\text { Theoretical } \\
\text { calculation from } \\
\beta^{\text {unp }}\end{array}$} & \multicolumn{2}{|c|}{$\begin{array}{c}\text { Theoretical } \\
\text { calculation from } \\
\beta^{-1}\end{array}$} \\
\hline & $\begin{array}{c}\text { Tilt angle } \\
\left({ }^{\circ}\right)\end{array}$ & $\varepsilon_{e}^{\mathrm{a}}$ & $\begin{array}{c}\text { Tilt angle } \\
\left({ }^{\circ}\right)\end{array}$ & $\varepsilon_{e}$ & $\begin{array}{c}\text { Tilt angle } \\
\left({ }^{\circ}\right)\end{array}$ & $\varepsilon_{e}$ \\
\hline & & & -4.3 & & -4.5 & 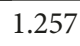 \\
\hline 12.2 & & & -7.5 & 10 & -7.6 & 1.24 \\
\hline 11.5 & -1 & & -12.6 & 1.2 & -12.8 & 1.23 \\
\hline 10.95 & -17.4 & 1.207 & -16.8 & 1.229 & -17.1 & 1.21 \\
\hline
\end{tabular}

${ }^{\mathrm{a}} \varepsilon_{e}$ is the equivalent permittivity of the element shown in Figure 3.

simulation is shown in Table 1 . We can see that the computation considering the perturbation of slots is in good agreement with that of the full-wave simulation. Meanwhile, the computation without considering slots has a larger error. It verifies the theoretical model ( 8 and 9 ) is accurate and significant in supplementing (6). Besides, the higher the frequency, the higher the consistency. There are little computational differences at higher frequencies, which may be due to the frequency-independent approximation of the impedance $Z s$ or the larger operating wavelength, the larger truncation error.

\section{Research on Nonlinear Design of Rectangular Grating of Slow-Wave Structure}

As a leaky-wave antenna, the periodic design makes the radiation power of each gap to decrease. Both the variation of CTS layer and SWS design can affect radiation power of each element. Considering the difficulty of design and fabrication, we keep the CTS layer of each element the same, then controlling the radiation power of each element by 


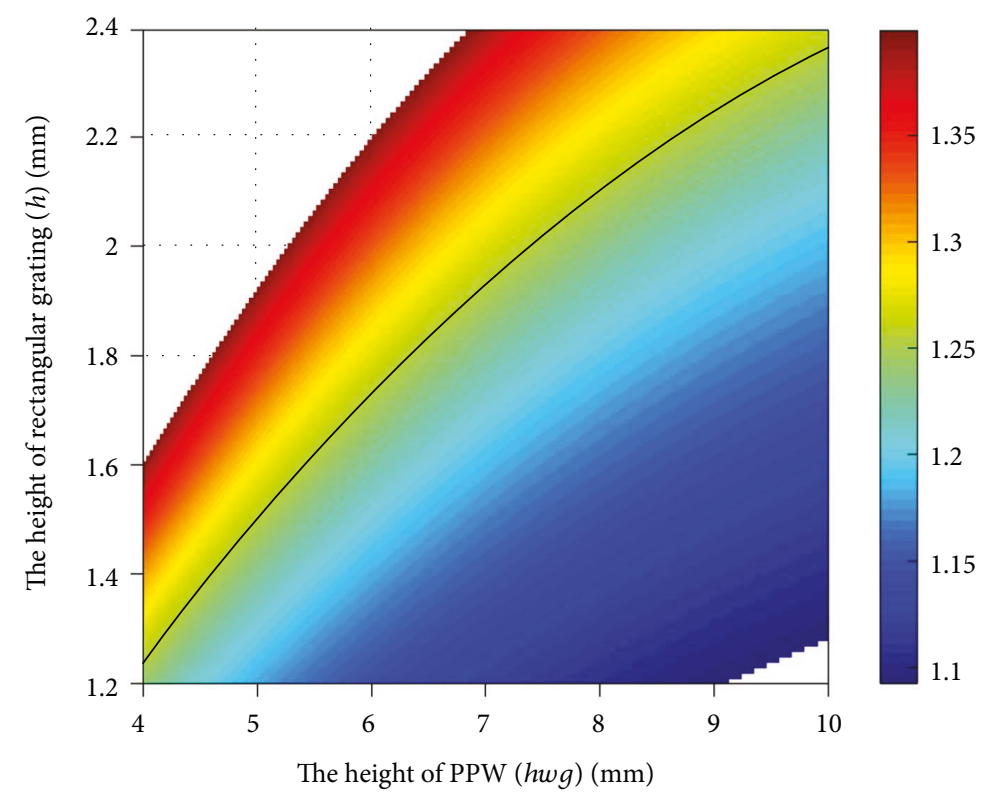

Figure 7: The relation among $\varepsilon_{e}, h$, and $h w g$.

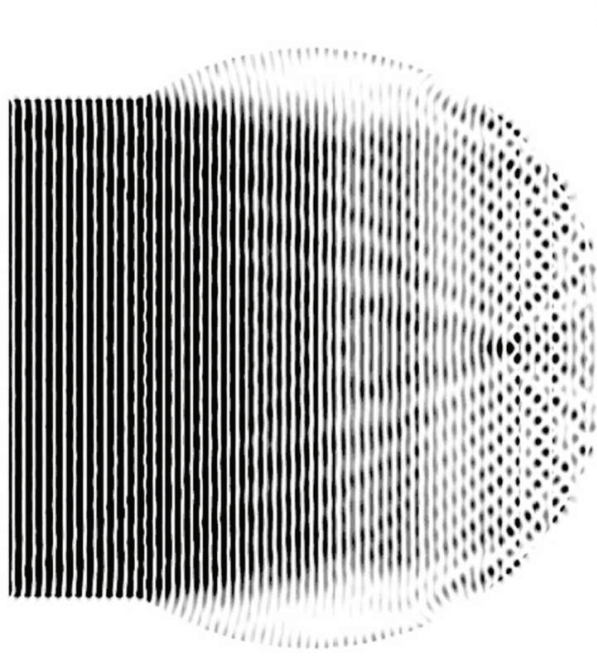

(a)

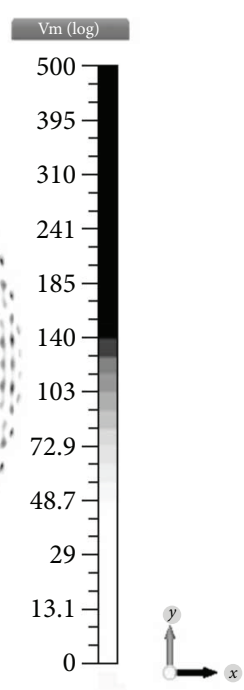

Figure 8: Comparison of electric field intensity in PPW at $12.5 \mathrm{GHz}$ before and after double nonlinear design of $h$ and $h w g$. (a) Constant $h w g$, constant $h$, (b) nonlinear $h w g$, and nonlinear $h$.

nonlinearly decreasing $h w g$ as the propagation distance increases in the $\mathrm{X}$ direction (hereinafter called "nonlinear $h w g$ " for short). We can design a nonlinear hwg to control the main lobe width and sidelobe level of the pattern. But the nonlinear $h w g$ is an extra research topic and will not be introduced. In this paper, in order to control the radiation energy as uniform as possible to improve gain of the antenna, we have already design an appropriate nonlinearity in $h w g$. On the basis of this nonlinear $h w g$, we research on keeping the equivalent permittivity of each element constant by designing the nonlinear rectangular grating SWS.

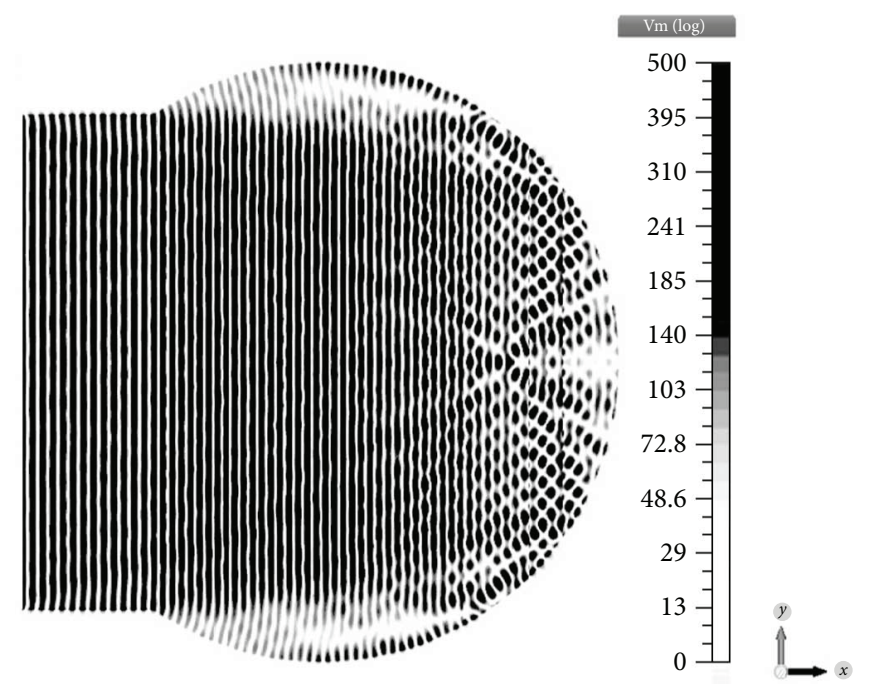

(b) 


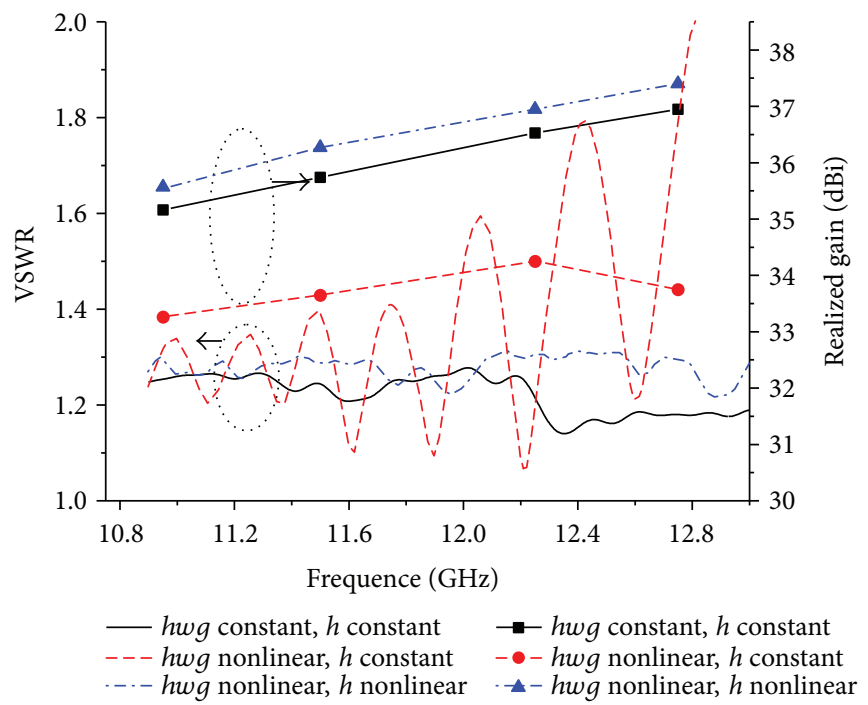

FIGURE 9: Full-wave simulation results of three different VICTS antennas.

the magnitude of $\varepsilon_{e}$. The black curve in Figure 7 is a polynomial curve obtained by fitting discrete points that are same in $\varepsilon_{e}$. Thus, points $(h w g, h)$ picked from the curve can maintain the uniformity of the equivalent permittivity of each element.

Hereto, all parameters of the model have been fixed and electromagnetic simulation is done. We design a prototype of $700 \mathrm{~mm}$ circular-aperture VICTS antenna with slopeshaped PPW and SWS shown in Figures 1(a) and 1(b). Figure 8 is a comparison of electric field intensity in PPW before and after double nonlinear design. The electric field of the latter part in the $\mathrm{X}$ direction enhances and its distribution is more uniform. Finally, we compare VSWR and realized the gain of VICTS antenna with three different conditions and results are shown in Figure 9. Condition 1, constant $h$ and constant hwg (i.e., the antenna shown in Figure 5), is represented by black broken line and black curve; condition 2, constant $h$ and nonlinear $h w g$, is represented by red broken line and red curve; condition 3 relatively varies between $h$ and $h w g$ (i.e., the antenna shown in Figure 1), is represented by blue broken line and blue curve. Broken lines with symbols are showing the realized gain of the antenna, while curves are showing VSWR of the antenna. The blue line is significantly higher than black line while red line and red curve obviously deteriorate. It verifies that it is both nonlinear $h$ and nonlinear $h w g$ but it is not singly nonlinear hwg causing the optimization. Meanwhile, it proves practicability and validity of the theoretical model in VICTS antenna design.

\section{Conclusion}

The theoretical model for rectangular grating SWS of VICTS element is established by combining the study of the semiopen rectangular grating SWS in the PPW and equivalent circuit model of leaky-wave antenna. Through comparing the computation of the theoretical model with the full-wave simulation, the validity and accuracy of the model are verified. Furthermore, by applying the theoretical model, difficulty in keeping the propagation constant in the same in the double nonlinear design of VICTS element is solved. By means of the double nonlinear design, VICTS antenna improves $0.4 \mathrm{~dB}$ gain. The paper provides a strong theoretical tool for analyzing and designing SWS of VICTS antenna.

\section{Conflicts of Interest}

The authors declare that they have no conflicts of interest.

\section{References}

[1] E. L. Holzman, "Pillbox antenna design for millimeter-wave base-station applications," IEEE Antennas and Propagation Magazine, vol. 45, no. 1, pp. 27-37, 2003.

[2] M. Ettorre, F. F. Manzillo, M. Casaletti, R. Sauleau, L. le Coq, and N. Capet, "Continuous transverse stub array for Ka-band applications," IEEE Transactions on Antennas and Propagation, vol. 63, no. 11, pp. 4792-4800, 2015.

[3] W. W. Milroy, "Continuous transverse stub element devices and methods of making same," US Patent US5266961A, 1993.

[4] W. W. Milroy, S. B. Coppedge, and A. C. Lemons, "Variable inclination continuous transverse stub array," US Patent US6919854, 2005.

[5] A. Sutinjo, M. Okoniewski, and R. H. Johnston, "Radiation from fast and slow traveling waves," IEEE Antennas and Propagation Magazine, vol. 50, no. 4, pp. 175-181, 2008.

[6] Z. Li, Research on the Radiation Mechanism of TravelingWave Structure and Novel Leaky-Wave Antennas, Diss. Beijing Jiaotong University, 2012, http://kns.cnki.net/KCMS/detail/ detail.asp $x$ ?dbcode $=$ CDFD\&dbname $=$ CDFD1214\&filename $=$ 1013135812.nh\&v=Mjc3NjlPZHVGeXptV3JyQVZGMjZIYks 3RzluTnJaRWJQSVI4ZVgxTHV4WVM3RGgxVDNxVHJXT TFGckNVUkxLZlo=.

[7] Y. Huang, Analysis of Open Rectangular Grating Sws, Ph.D. dissertation, University of Electronic Science and Technology 
of China, 2007, http://kns.cnki.net/KCMS/detail/detail.aspx? dbcode $=$ CMFD\&dbname $=$ CMFD2008\&filename $=200705075$ 9.nh\&v=MDk5MDRHYk85SHRiSnBwRWJQSVI4ZVgxTHV 4WVM3RGgxVDNxVHJXTTFGckNVUkxLZlpPZHVGeXpu Vjd6QVYxMjc=.

[8] F. J. Garcia-Vidal, L. Martin-Moreno, and J. B. Pendry, "Surfaces with holes in them: new plasmonic metamaterials," Journal of Optics A: Pure and Applied Optics, vol. 7, no. 2, pp. S97-S101, 2005.

[9] G. Valerio, Z. Sipus, A. Grbic, and O. Quevedo-Teruel, "Nonresonant modes in plasmonic holey metasurfaces for the design of artificial flat lenses," Optics Letters, vol. 42, no. 10, pp. 2026-2029, 2017.

[10] P. Burghignoli, G. Lovat, and D. R. Jackson, "Analysis and optimization of leaky-wave radiation at broadside from a class of 1-D periodic structures," IEEE Transactions on Antennas and Propagation, vol. 54, no. 9, pp. 2593-2604, 2006. 


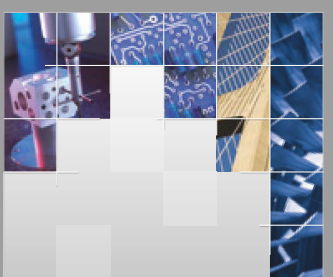

\section{Enfincering}
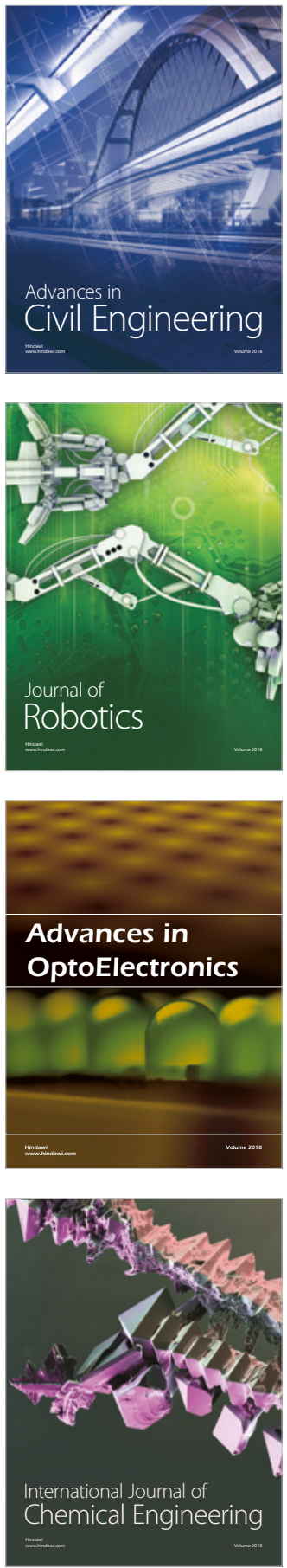

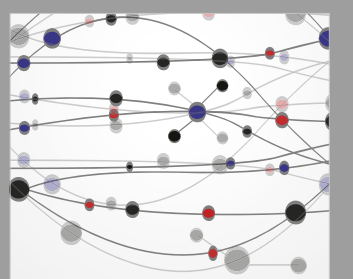

\section{Rotating \\ Machinery}

The Scientific World Journal

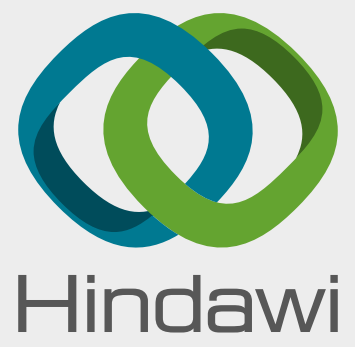

Submit your manuscripts at

www.hindawi.com
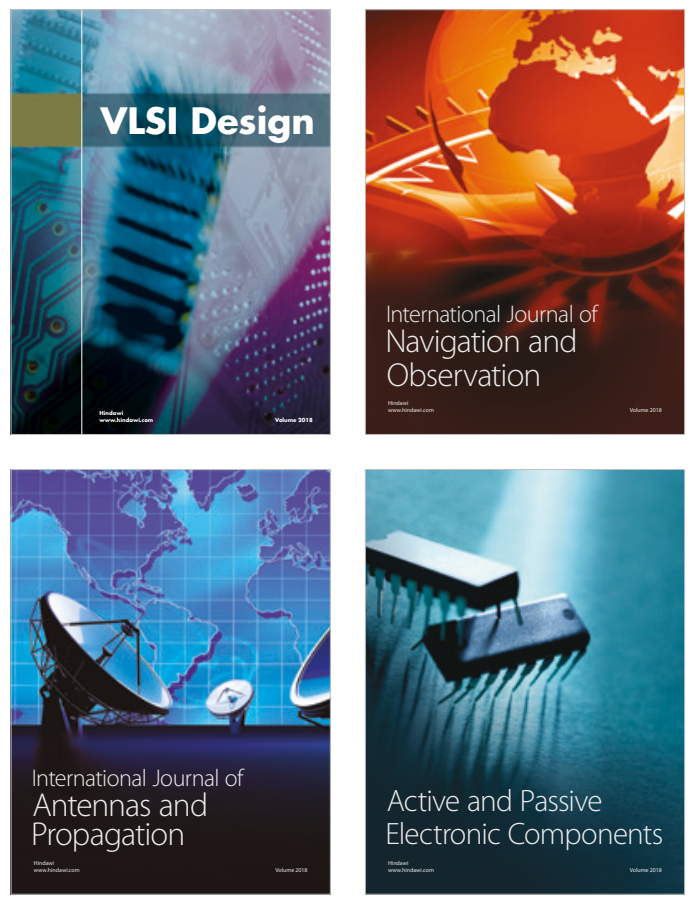
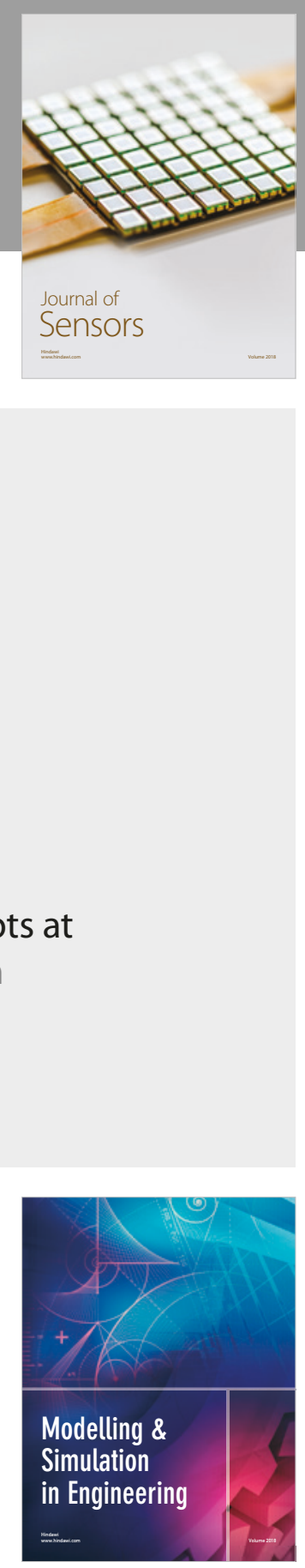

\section{Advances \\ Multimedia}
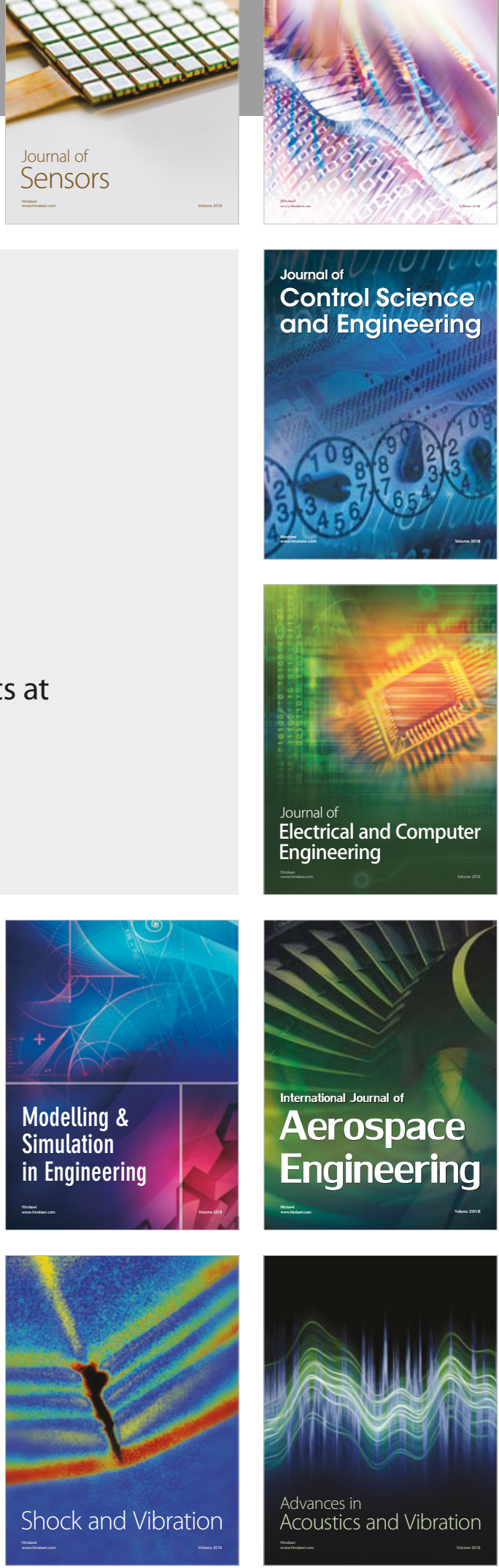